\title{
Effect of Fc Fusion on Folding and Immunogenicity of Middle East Respiratory Syndrome Coronavirus Spike Protein
}

\author{
Jungmin Chun ${ }^{1,2 \dagger}$, Yeondong Cho ${ }^{1,2 \dagger}$, Ki Hoon Park ${ }^{1,2}$, Hanul Choi ${ }^{1,2}$, Hansam Cho ${ }^{2}$, Hee -Jung Lee ${ }^{2}$, Hyun Jang ${ }^{3}$, \\ Kyung Hyun $\mathrm{Kim}^{4}$, Yu-Kyoung $\mathrm{Oh}^{5}$, and Young Bong Kim ${ }^{1,2 *}$ \\ ${ }^{1}$ Department of Bio-industrial Technologies, Konkuk University, Seoul 05029, Republic of Korea \\ ${ }^{2}$ Department of Biomedical Science and Engineering, Konkuk University, Seoul 05029, Republic of Korea \\ ${ }^{3}$ WooGene BEG, Seoul, 07299, Republic of Korea \\ ${ }^{4}$ Department of Biotechnology and Bioinformatics, Korea University, Sejong 30019, Republic of Korea. \\ ${ }^{5}$ College of Pharmacy and Research Institute of Pharmaceutical Sciences, Seoul National University, Seoul 08826, Republic of Korea
}

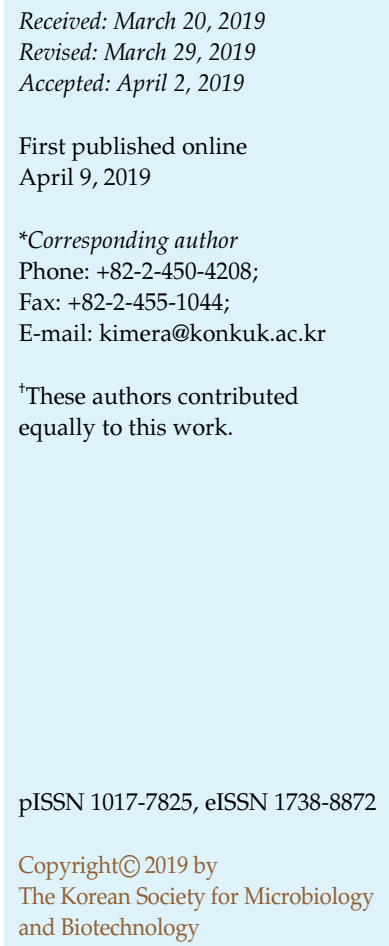

\begin{abstract}
Middle East respiratory syndrome coronavirus (MERS-CoV) induces severe respiratory impairment with a reported mortality rate of $\sim 36 \%$ in humans. The absence of clinically available MERS-CoV vaccines and treatments to date has resulted in uncontrolled incidence and propagation of the virus. In vaccine design, fusion with the IgG Fc domain is reported to increase the immunogenicity of various vaccine antigens. However, limited reports have documented the potential negative effects of Fc fusion on vaccine antigens. To determine whether Fc fusion affects the immunogenicity of MERS-CoV antigen, we constructed a Fcassociated MERS-CoV spike protein (eS770-Fc, $110 \mathrm{kDa}$ ), whereby human IgG4 Fc domain was fused to MERS-CoV spike protein (eS770) via a Gly/Pro linker using baculovirus as the expression system. For comparative analyses, two eS770 proteins lacking the IgG4 Fc domain were generated using the IdeS protease $(\mathrm{eS} 770-\Delta \mathrm{Fc})$ or His tag attachment (eS770-His) and the immunogenicity of the above constructs were examined following intramuscular immunization in mice. Contrary to expectations, non-Fc spike proteins (eS770- $\Delta \mathrm{Fc}$, eS770-His; $90 \mathrm{kDa}$ ) showed higher immunogenicity than the Fc fusion protein (eS770-Fc). Moreover, unlike nonFc spike proteins, eS770-Fc immunization did not elicit neutralizing antibodies against MERS$\mathrm{CoV}$. The lower immunogenicity of Fc-fused eS770 was related to alterations in the structural conformation of the spike protein. Taken together, our results indicate that IgG Fc fusion reduces the immunogenicity of eS770 by interfering with the proper folding structure.
\end{abstract}

Keywords: MERS-CoV, subunit vaccine, IgG Fc domain, protein folding, immunogenicity

\section{Introduction}

Middle East respiratory syndrome coronavirus (MERS$\mathrm{CoV}$ ) is a beta coronavirus with an enveloped singlestranded, positive-sense RNA genome [1] encoded by ORF1a, 1ab (by frameshifting), 3, 4a, 4b, 5 and 8b, along with genes for spike (S), envelope (E), matrix (M) and nucleocapsid $(\mathrm{N})$ proteins [2]. Spike is a major viral protein of MERS-CoV that binds dipeptidyl peptidase 4 (DPP 4) to facilitate viral entry. Owing to the distribution of DPP 4 in human endothelial and epithelial cells, MERS-CoV infection in humans causes acute respiratory diseases, such as severe acute respiratory syndrome (SARS) [3].

MERS-CoV infection is fatal and pandemic. The World Health Organization reported 790 deaths among 2,220 cases (35.6\% mortality) from 2012 to May 2018 [4]. To date, MERS$\mathrm{CoV}$ has been detected in 24 countries, and associated with high mortality rates [5]. In 2015, an outbreak of MERS-CoV in Korea caused 38 deaths among 186 registered cases [6].

Given the clinical significance of MERS-CoV infection and disease, the need for preventive vaccines and curative treatments remains an urgent unmet medical issue. While a 
number of attempts have been made, further research is essential to develop effective MERS-CoV vaccines that induce high titers of neutralizing antibodies.

One of the strategies used to design subunit vaccines with enhanced immunogenicity is linkage with the IgG Fc domain based on previous reports that genetic fusion of this domain to vaccine antigens promotes antibody induction and neutralizing activity $[7,8]$ in addition to altering antigen pharmacokinetics. Decreased renal clearance by the antigen due to dimerization by the Fc domain has been reported [9]. Extended half-life has been demonstrated through interactions with a neonatal Fc receptor (FcRn) [10].

Here, we constructed a MERS-CoV spike protein, eS770, both without and in combination with human IgG4 Fc (eS770-Fc), with a view to examining the effect of the Fc fusion on folding and immunogenicity of the MERS-CoV subunit vaccine antigen.

\section{Materials and Methods}

\section{Cells}

Sf9 cells were cultured in SF-900 II medium (Gibco, Waltham, Massachusetts, USA) containing $1 \%$ antibiotic-antimycotic solution (Gibco) at $27^{\circ} \mathrm{C}$. High Five cells were cultured at $27^{\circ} \mathrm{C}$ in Express Five medium containing $90 \mathrm{ml} \mathrm{L-glutamine} \mathrm{(200} \mathrm{mM)} \mathrm{(Gibco)} \mathrm{and}$ $1 \%$ antibiotic-antimycotic solution. 293T and Huh7 cells were cultured in DMEM (Gibco) supplemented with 10\% heatinactivated fetal bovine serum and $1 \%$ penicillin/streptomycin (Gibco) at $37^{\circ} \mathrm{C}$ under $5 \% \mathrm{CO}_{2}$.

\section{Animals}

Female BALB/c mice (6 weeks old) (Orient-Bio, Korea) were provided water and fed ad libitum in the animal facility at the College of Animal Bioscience and Technology, Konkuk University. Experimental procedures were approved by the Konkuk University Institutional Animal Care and Use Committee (IACUC Approval No: KU17021).

\section{Construction of Plasmid DNA Encoding eS770-Fc}

For generation of plasmid DNA encoding eS770-Fc, the MERS$\mathrm{CoV}$ spike partial gene was genetically fused to the human IgG4 Fc domain through the Gly/Pro linker. The Fc-fused spike partial gene was linked to a baculovirus gp64 signal sequence for expression of the fusion protein outside the cell under control of the polyhedrin promoter $\left(\mathrm{P}_{\mathrm{PH}}\right)$ of pFastBac 1 vector. All gene constructs used in the cloning procedure were approved by Korea Centers for Disease Control Prevention (Approval No: 16-RDM008) and custom-synthesized by Bioneer Ltd. (Korea). All the synthesized genes were confirmed via restriction enzyme digestion and sequencing.
Preparation and Amplification of Recombinant Baculovirus Encoding eS770-Fc

To prepare recombinant baculovirus (rBV) expressing the above plasmid constructs, recombinant Bacmid was generated using the Bac-to-Bac Expression System (Invitrogen, USA) and transfected into Sf9 cells using Cellfectin II reagent (Gibco). The supernatant containing rBV was harvested three days after transfection and used as P0 viral stock for infection (0.01 MOI) into static T-flasks $\left(25 \mathrm{~cm}^{2}\right.$ (P1) or $75 \mathrm{~cm}^{2}$ (P2)) and roller bottles (P3). Three days after infection, cells were harvested via centrifugation at $6,760 \times g$ for $10 \mathrm{~min}$ and the supernatant used as seed virus. Virus titer was measured using the BakPak Baculovirus Rapid Titer Kit (Clontech, Mountain View, USA).

\section{Expression and Characterization of eS770-Fc}

Recombinant proteins were expressed using the baculovirus/ insect cell expression system. In brief, the High Five cell line was infected with three multiplicity of infection (MOI) of rBVs (Ac eS770-Fc). The supernatant was harvested $72 \mathrm{~h}$ after infection and centrifuged at $6,760 \mathrm{xg}$ for $10 \mathrm{~min}$. The harvested eS770-Fc protein was equilibrated using buffer (100 mM sodium phosphate, $1.5 \mathrm{M}$ $\mathrm{NaCl}, \mathrm{pH} 8.0$ ) and centrifuged at $18,800 \times g$ for $20 \mathrm{~min}$. The resulting supernatant was collected and run through a protein $\mathrm{A}$ agarose affinity chromatography column (Thermo Fisher, USA) for purification of recombinant protein $(\mathrm{eS} 770-\mathrm{Fc})$. Concentrated and purified eS770-Fc protein was confirmed via Coomassie blue staining and western blot using a goat anti-MERS Spike antibody (SICGEN, Portugal). Band density was analyzed using MultiGauge Software (Fuji Photo Film Co. Ltd., Japan).

\section{Size Exclusion Chromatography (SEC)}

The eS770-Fc protein obtained via protein A affinity chromatography was further purified using SEC. In brief, the protein $(1.2 \mathrm{mg} / \mathrm{ml})$ was loaded on a Superose $610 / 300 \mathrm{GL}$ prepacked column (GE Healthcare, Chicago, Illinois, USA) under a flow rate of $0.5 \mathrm{ml} / \mathrm{min}$. Absorbance of the eluting protein was spectrophotometrically measured at $280 \mathrm{~nm}$ and purity of the elution fraction determined via SDS-PAGE. Purified recombinant proteins were stored at $-80^{\circ} \mathrm{C}$ until use.

\section{Preparation of eS770 Lacking Fc}

For preparing eS770 devoid of $F_{c}(e S 770-\Delta F c)$, eS770-Fc was treated with IdeS protease (Promega, USA) at $37^{\circ} \mathrm{C}$ for $1 \mathrm{~h}$ for cleavage of the IgG Fc domain. The eS770- $\triangle \mathrm{Fc}$ was purified through a protein A agarose affinity chromatography. The Fc part of the protein or uncut eS770-Fc were removed using a protein A agarose, and the unbound $\mathrm{eS770}-\Delta \mathrm{Fc}$ was harvested. For the animal experiment, the eluted eS770- $\Delta \mathrm{FC}$ was concentrated using Vivaspin Turbo 15 (Germany). In other experiments, eS770 without Fc was prepared by fusion to a histidine tag (eS770-His). The eS770-His protein was expressed in High Five cells and purified using TALON Metal Affinity Resin (Clontech, USA). 


\section{Mouse Immunization}

Three groups of six-week-old female SPF BALB/c mice (5 per group) were immunized with PBS (G1), $1 \mu \mathrm{g}$ of each recombinant protein eS770-Fc (G2) or eS770- $\Delta \mathrm{Fc}$ (G3) with alum adjuvant (Thermo Fisher). Immunization was performed intramuscularly with two-week intervals. Blood samples were collected from mouse cheek 10 days after each immunization (days 10 and 24)

\section{Enzyme-Linked Immunosorbent Assay (ELISA)}

Indirect ELISA was used to determine the levels of serum antibodies specific for MERS-CoV spike protein. For ELISA plate coating, purified MERS-CoV spike protein expressed in $\mathrm{CHO}$ cells supplied by WooGene B\&G (Korea) was added to 96-well plates (Nunc, Roskilde, Denmark) and incubated overnight at $4^{\circ} \mathrm{C}$. After blocking with $2 \%$ bovine serum albumin (BSA) in PBS, serum samples diluted 1:300 (for IgG) with PBS were added into wells and incubated for $2 \mathrm{~h}$. Horseradish peroxidase (HRP)-labeled goat anti-mouse IgG $(2 \mathrm{mg} / \mathrm{ml}$; Abcam, UK) diluted with PBS containing $0.025 \%$ Tween-20 and 1\% BSA (diluted 1:10000) was pipetted into the plate and incubated for $1 \mathrm{~h}$ at room temperature. ELISA was performed with a 3,3,5,5-tetramethylbenzidine (TMB) solution (Life Technologies, USA). The reaction was terminated by adding $1 \mathrm{~N} \mathrm{H}_{2} \mathrm{SO}_{4}$. Absorbance at $450 \mathrm{~nm}$ was measured using a microplate reader (BioTek, USA) and the results expressed as reciprocal numbers of the final detectable dilution.

\section{Neutralization Assay with Pseudotype Virus (PV)}

MERS-CoV pseudotyped virus was prepared for the neutralization assay as reported previously [11]. The pcDNA3.1 (+)-MERS Spike construct was provided by Dr. Shibo Jiang of Fudan University (China). pNL4-3 Luc R-E- and pcDNA3.1(+)MERS spike were co-transfected to 293T cells using iN-fect in vitro transfection reagent (iNtRON, Korea). Expression of PV was confirmed via western blot. After infection of Huh7 cells with PV, titration was performed using the luciferase assay.

For the luciferase-based neutralization assay, blood samples were initially incubated at $56^{\circ} \mathrm{C}$ for $30 \mathrm{~min}$ for complement heat inactivation, followed by two-fold serial dilution (1:40 to 1:20480) using serum-free DMEM. Pseudovirus-containing media $\left(5 \times 10^{3} \mathrm{RLU} / 50 \mu \mathrm{l}\right)$ were incubated with diluted serum $(50 \mu \mathrm{l})$ at $37^{\circ} \mathrm{C}$ for $1 \mathrm{~h}$ before adding to Huh 7 cell in 96-well culture plates. After incubation at $37^{\circ} \mathrm{C}$ for $48 \mathrm{~h}$, the supernatant was removed and washed three times with PBS. Relative light unit (RLU) was measured using Beetle Lysis-Juice (PJK GmbH, Cat No. 102512) and a Luminescence Counter (PerkinElmer, USA) according to the manufacturer's instructions. The measured RLU is calculated by the following equation to obtain the neutralizing activity.

Neutralizing activity $=100-\left(\frac{R L U \text { of serum sample }}{R L U \text { of control }}\right) \times 100$

The neutralizing antibody titer was determined as a dilution factor of the antibody until $\geq 50 \%$ decrease in RLU.

\section{Statistical Analysis}

All statistical analyses were performed using GraphPad 7.0 software (USA) and data presented as means \pm standard error of mean (SEM). For analysis of the statistical significance of differences, one-way or two-way analysis of variance (ANOVA) was applied. $P$ values $\leq 0.05$ were considered statistically significant.

\section{Results}

\section{Expression and Characterization of Recombinant Protein eS770 with or without $\mathrm{Fc}$}

To determine the effects of Fc fusion, we generated MERS-CoV partial spike protein constructs with or without the Fc region. Part of the spike protein was synthesized

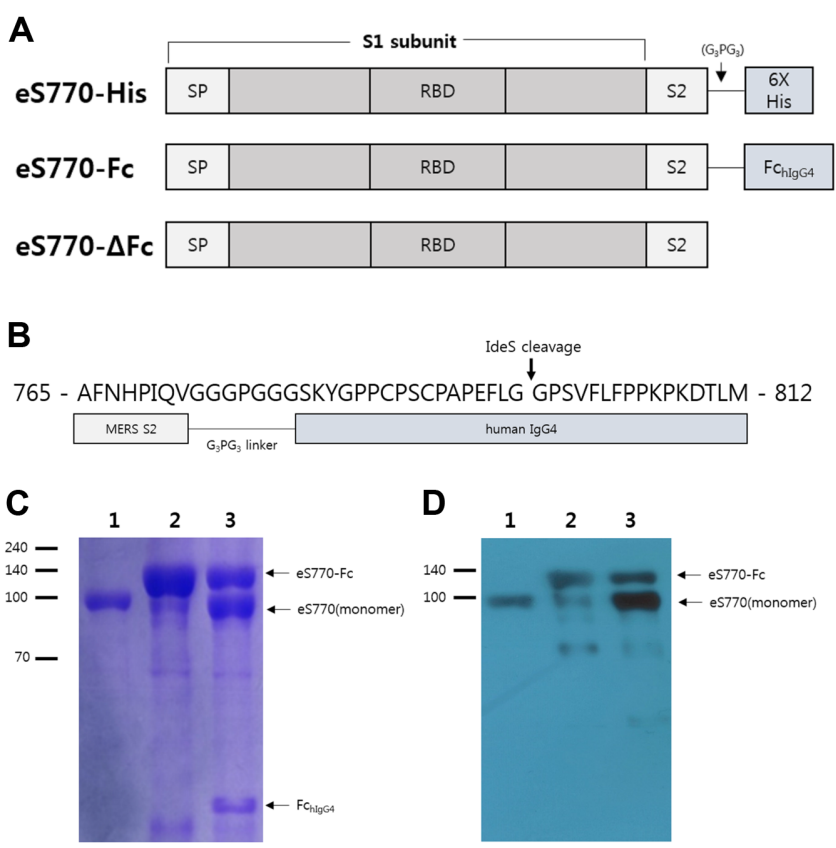

Fig. 1. Characterization of recombinant MERS spike proteins. (A) Schematic representation of with or without Fc-linked and Histagged MERS spike proteins. Protein constructs consist of the S2 partial truncated MERS-CoV spike protein codon optimized in the insect cell expression system. MERS-CoV spike protein is fused to the human IgG4 Fc fragment and histidine tag through a Gly-Pro linker. (B) Amino acid sequence cleaved by IdeS protease. The number indicates amino acids. SP: Autographa californica nucleopolyhedrovirus $(A c M N P V)$ gp64 signal peptide; RBD: receptor-binding domain. (C) SDS-PAGE analysis of affinity chromatography-purified forms of recombinant spike protein. Proteins were resolved via 10\% SDSPAGE under reducing conditions and stained with Coomassie brilliant blue. 1: eS770-His 2: eS770-Fc, 3: eS770- $\Delta \mathrm{Fc}_{\mathrm{C}}$ (Fc deleted with IdeS). (D) Western blot illustrating expression of spike protein upon probing with an anti-MERS CoV spike antibody. The spike protein formed monomers under reducing conditions. Samples were resolved via $10 \%$ SDS-PAGE. 1: eS770-His 2: eS770-Fc, 3: eS770- $\Delta$ Fc with IdeS. 
with receptor binding domain that was codon-optimized in insect cells. Human IgG4 Fc domain was fused to a Gly/ Pro linker and the MERS signal sequence replaced with the baculovirus gp64 signal sequence (Fig. 1A). For baculovirus/ insect cell expression of eS770-Fc, Sf9 cells were transfected with the generated recombinant bacmid. High Five cells were infected with recombinant baculovirus and the secreted recombinant eS770-Fc purified. To prepare eS770 lacking $\mathrm{Fc}$, the $\mathrm{Fc}$ region was enzymatically removed as illustrated in (Fig. 1B). Expression of recombinant protein was detected via SDS-PAGE and Coomassie brilliant blue staining as well as western blot. In Coomassie staining, Unlike lines 1 and 2, line 3 is eS770- $\Delta$ Fc after IdeS protease treatment. As is shown, IdeS did not completely cleave the protein, so eS770 monomer and undigested eS770-Fc proteins appeared together (Fig. 1C).

Western blot confirmed the presence of eS770 protein in both eS770-Fc and eS770- $\Delta$ Fc samples. A major band was detected in the molecular weight region of the eS770 monomer (Fig. 1D). We further compared western blot (Fig. 1D) and Coomassie brilliant blue staining (Fig. 1C) band densities using Multi-gauge software (Fuji Photo Film Co. Ltd., Japan). In the case of eS770-Fc, a weaker band was detected in the western blot than Coomassie stain (Coomassie blue 72.9: western blot 39.8) while for the eS770 monomer band of eS770- $\Delta \mathrm{Fc}$, a relatively strong band on the western blot (Coomassie blue 47.78: western blot 74.31) was observed.

\section{Effect of Fc Fusion on Induction of Spike Protein-Specific IgG Antibody}

To determine the effects of Fc fusion on immunogenicity, mice were immunized with eS770-Fc, eS770- $\Delta \mathrm{Fc}$ or PBS. Immunization and sample collection schedules are presented in Fig. 2A and injection route, dose and adjuvant in Fig. 2B. In the PBS treatment group, spike proteinspecific IgG antibody in serum was not detected. In groups treated with eS770-Fc and eS770- $\Delta \mathrm{Fc}$, spike protein-specific IgG antibody titers were increased more than an order of magnitude at 24 days after the first immunization, compared to that at 10 days post-immunization (Fig. 2C). Notably, however, the eS770- $\Delta \mathrm{Fc}$ treatment group showed significantly greater titers of spike protein-specific IgG antibody, compared with the eS770-Fc treatment group.

\section{Effect of Fc Fusion on Induction of Neutralizing Antibody}

Next, we examined whether the fusion with Fc negatively affects induction of the neutralizing antibody using MERS PV to perform the neutralization assay. In vaccine studies, virus-neutralizing antibody measurement is a very important indicator. To do this, live MERS-CoV should be used to test for neutralizing antibody activity in the BL3 facility. However, MERS-CoV pseudotyped virus was constructed for simple and safe neutralizing antibody measurement in the BL2 facility. Fusion with Fc clearly affected the efficacy of induction of the neutralizing antibody. In the group treated with eS770-Fc, negligible
A

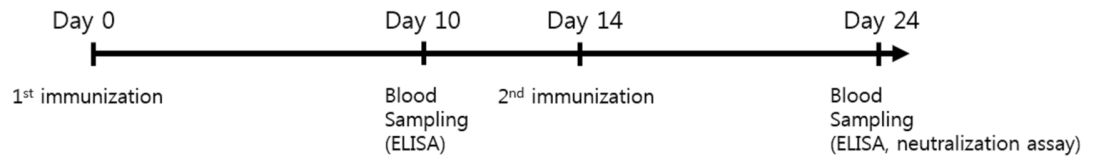

B

\begin{tabular}{|l|l|l|l|l|}
\hline GROUP & INJECTION & ROUTE & VOLUME & AMOUNT \\
\hline 1 & PBS & I.M. Right \& Left thigh & $100 \mathrm{ul}$ & $100 \mathrm{ul}$ \\
\hline 2 & eS770-FC & I.M. Right \& Left thigh & $100 \mathrm{ul}$ & $1 \mathrm{ug} / \mathrm{mice}$ with Alum adjuvant \\
\hline 3 & eS770- $\triangle \mathrm{FC}$ & I.M. Right \& Left thigh & $100 \mathrm{ul}$ & $1 \mathrm{ug} /$ mice with Alum adjuvant \\
\hline
\end{tabular}

C

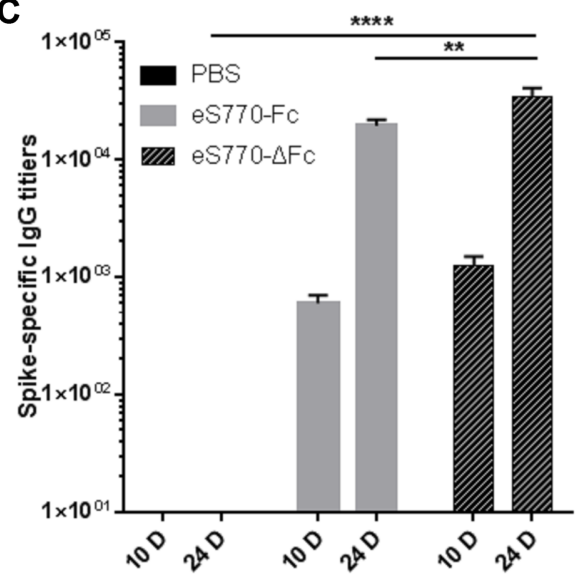

Fig. 2. Recombinant Fc-depleted spike proteins are more immunogenic than Fc-fused proteins in the presence of adjuvants.

(A) Diagram of immunization schedules. Mice were boosted on week 2 via the I.M. route. Serum was collected 10 days after every immunization. (B) Mice were divided into three groups and immunized using recombinant spike proteins with Alum adjuvant. Control mice were injected with PBS. (C) Mice (five per group) were immunized I.M. with $1 \mu \mathrm{g}$ recombinant protein in Alum adjuvant. Data represent mean values \pm SEM. Data were considered significant at ${ }^{* *} p<0.01$ and ${ }^{* * * *} p<0.0001$ (two-way ANOVA). 

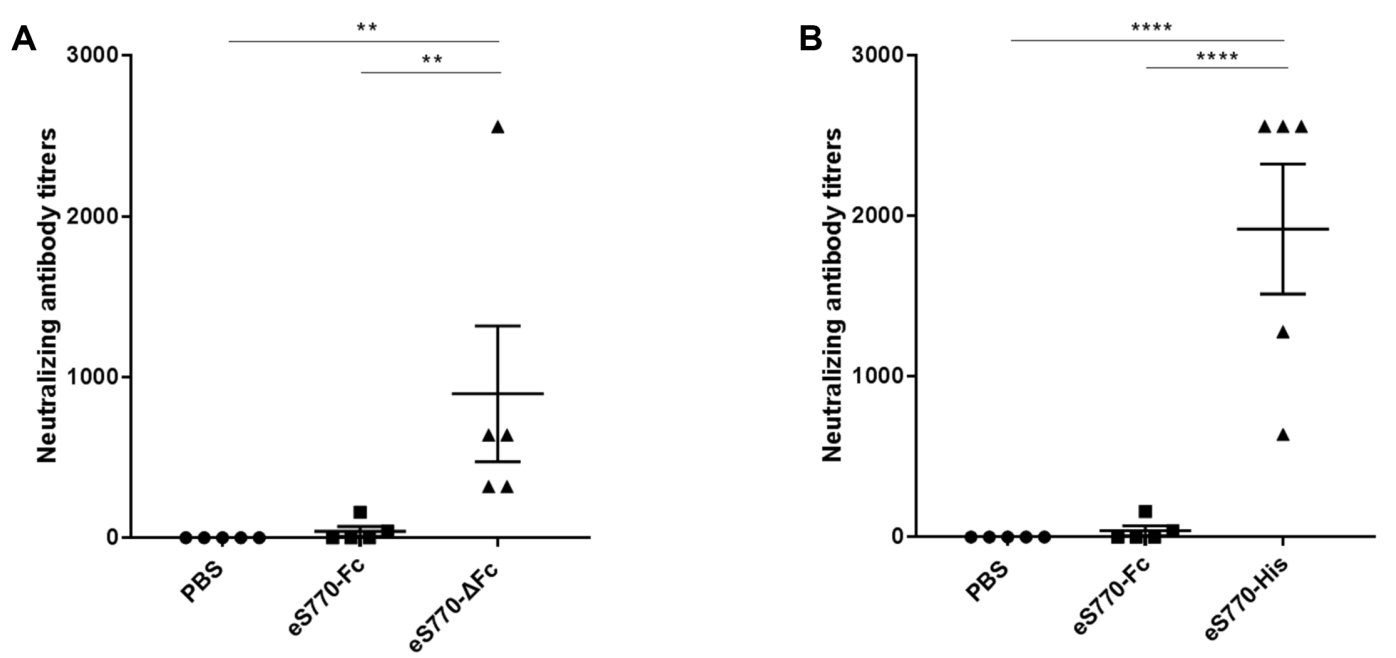

Fig. 3. MERS-CoV-specific neutralization antibody titers in immunized mice.

Serum samples were collected on day 10 after the second immunization to determine neutralization antibody titers. (A) Comparison of neutralizing antibody titers between eS770-Fc and eS770- $\Delta$ Fc. (B) Comparison of neutralizing antibody titers between eS770-Fc and eS770-His. Data are presented as means \pm SEM. Data were considered significant at ${ }^{* *} p<0.01$ and ${ }^{* * *} p<0.0001$ (one-way ANOVA).

neutralizing antibody was detectable, with an average neutralization titer of 40 . In contrast, eS770- $\Delta \mathrm{Fc}$ induced neutralizing antibody in $100 \%$ mice with an average neutralization titer of 896, signifying $>20$-fold increase (Fig. 3A).

To ascertain whether $\mathrm{F}_{\mathrm{C}}$ deletion contributes to alterations in the neutralizing antibody response, we additionally constructed eS770-His using a baculovirus expression system. Consistent with data obtained using eS770- $\Delta \mathrm{Fc}$,
eS770-His induced neutralizing antibody in 100\% mice. Moreover, immunization with eS770-His resulted in an average neutralization titer of 1920, indicating a 48 -fold increase relative to the group immunized with eS770-FC (Fig. 3B).

\section{Effect of Fc Fusion on eS770 Protein Aggregation}

Fusion with Fc affected physicochemical aggregation of the expressed protein. The SEC elution profiles disclosed
A

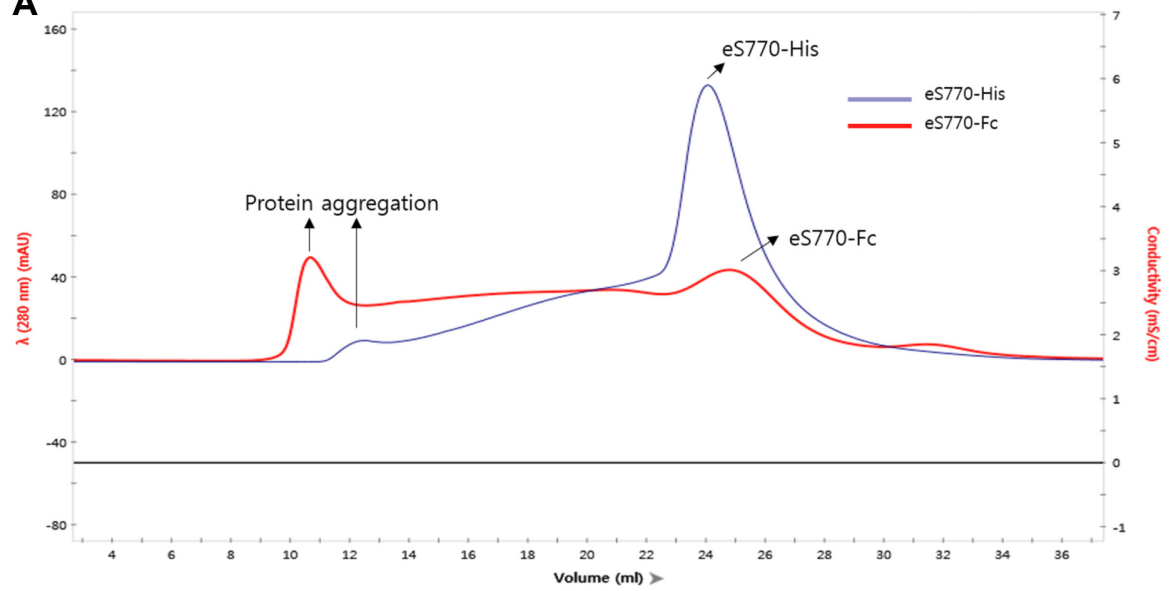

B

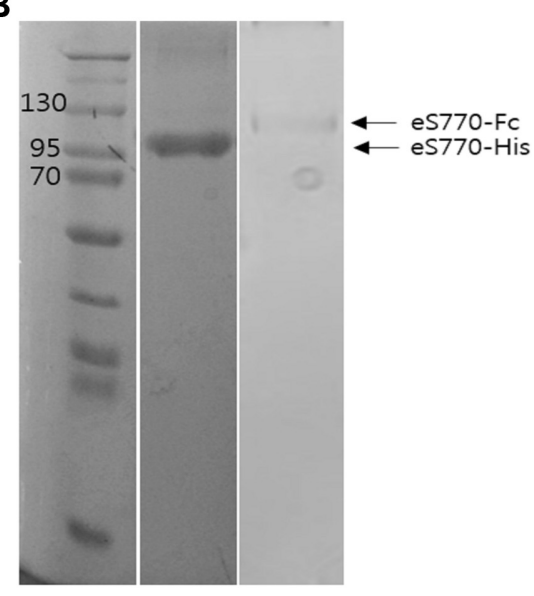

Fig. 4. Purification of the two recombinant proteins (eS770-His, eS770-Fc) from insect cell expression systems.

(A) Chromatogram showing the elution profile of the two recombinant proteins from a Superose 6 gel filtration column. (B) The fractions $(20 \mu \mathrm{l})$ corresponding to the peaks were mixed with $5 \mu \mathrm{l}$ of $5 \mathrm{X}$ sample buffer and loaded onto a 10\% SDS gel. eS770-His and eS770-Fc bands observed on the gel correspond to the expected sizes (1: eS770-His, 2: eS770-Fc). 
notable protein aggregation of eS770-Fc while eS770-His showed notably lower aggregation in SEC and higher concentrations of protein in the later fractions with elution volumes of 23 to $26 \mathrm{ml}$ (Fig. 4A). SDS-PAGE analysis of the later elution fractions of eS770-His disclosed the presence of a 90 kDa band while eS770-Fc displayed a band with a molecular weight of $\sim 110 \mathrm{kDa}$ (Fig. 4B).

\section{Discussion}

Our collective results suggest that genetic fusion with the IgG Fc region lowers the immunogenicity of MERS-CoV spike protein antigen. Fusion with Fc clearly hindered induction of MERS-CoV spike protein-specific serum IgG as well as neutralizing antibody against MERS PV.

The reduced immunogenicity of eS770 induced by fusion with Fc may be related to structural conformation changes

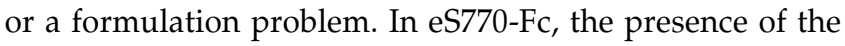
Fc moiety may contribute to multimer formation, leading to aggregation. As predicted, aggregate formation was observed in the SEC elution profile of eS770-Fc (Fig. 4A). In contrast to eS770-Fc, eS770-His did not form significant aggregates and showed a distinct structural peak.

The structural conformation of eS770 is crucial for the induction of immunogenicity. Previous studies have reported that MERS-CoV spike protein structurally exists as a trimer in the native state [12] while the Fc fusion protein is in a dimeric form [13]. In SEC elution profiles, the eS770-His peak was observed slightly earlier than the eS770-Fc peak, indicating a larger size of His-bound protein than eS770-Fc in the native form. If the eS770-Fc protein forms a trimer similar to a general spike protein, a larger-sized peak should be detected relative to the eS770His protein. However, based on the elution profile of eS770-Fc at later fractions, signifying smaller sizes, we assumed that the protein forms a dimer rather than a trimer, resulting in lower immunogenicity. Another way to determine effects on immunogenicity is to compare Coomassie brilliant blue staining and western blot results (Figs. 1C and 1D). In the case of eS770-Fc, the band from the western blot was weaker than the Coomassie blue stain while that of the eS770 monomer appeared relatively stronger than the Coomassie blue band. In view of these findings, we assume that the antibody binding capacity of eS770-Fc is hindered by Fc fusion to the spike protein.

We compared the effects of Fc-fused MERS-CoV spike protein with those of spike protein lacking the Fc region. In contrast to numerous reports of enhanced immunogenicity of Fc fusion in the design of vaccine antigens, our data show that non-Fc protein vaccine induces an even more potent immune response than Fc-fused vaccine, highlighting the importance of structural analysis after fusion with Fc. The alterations in structural conformation upon fusion with Fc should therefore be evaluated in advance of immunogenicity. According to paper 'MERSCoV Antibody Responses 1 Year after Symptom Onset, South Korea, 2015', the titers of neutralization antibody were from 10 to 320. Other MERS-CoV vaccine papers showed that the neutralization titer is usually 320 - 1280, which protect the MERS-CoV challenge [11, 14, 15]. Our neutralizing antibody data showed that eS770- $\Delta \mathrm{Fc}$ and eS770-His immunized group induced neutralization titer of 896 and 1920, respectively. These neutralization titers are enough to protect from MERS-CoV infection. In addition, It requires animal experiments using hDPP4-Tg mouse model to evaluate the actual protection ability of the recombinant MERS-CoV S protein (eS770-Fc, eS770- $\Delta \mathrm{Fc}$ eS770-His) vaccine against MERS-CoV in further study. Our findings provide insights that may aid in improving the design of $\mathrm{FC}_{\mathrm{C}}$ fusion vaccines in the future.

\section{Acknowledgments}

This work was supported by Konkuk University in 2016.

\section{Conflict of Interest}

The authors have no financial conflicts of interest to declare.

\section{Reference}

1. Alsolamy S, Arabi YM. 2015. Infection with Middle East respiratory syndrome coronavirus. Can. J. Respir. Ther. 51: 102.

2. Scobey T, Yount BL, Sims AC, Donaldson EF, Agnihothram SS, Menachery VD, et al. 2013. Reverse genetics with a fulllength infectious cDNA of the Middle East respiratory syndrome coronavirus. Proc. Natl. Acad. Sci. USA 110: 1615716162.

3. Wang C, Zheng X, Gai W, Zhao Y, Wang H, Wang $\mathrm{H}$, et al. 2017. MERS-CoV virus-like particles produced in insect cells induce specific humoural and cellular imminity in rhesus macaques. Oncotarget 8: 12686-12694.

4. Zhao G, He L, Sun S, Qiu H, Tai W, Chen J, et al. 2018. A novel nanobody targeting middle east respiratory syndrome coronavirus (MERS-CoV) receptor-binding domain has potent cross-neutralizing activity and protective efficacy against MERS-CoV. J. Virol. 92. 
5. Lee K, Ko HL, Lee E-Y, Park H-J, Kim YS, Kim Y-S, et al. 2018. Development of a diagnostic system for detection of specific antibodies and antigens against Middle East respiratory syndrome coronavirus. Microbiol. Immunol. 62: 574-584.

6. Kim S, Kim S. 2018. Exploring the determinants of perceived risk of Middle East respiratory syndrome (MERS) in Korea. Int. J. Environ. Res. Public Health 15(6).

7. Shubin Z, Li W, Poonia B, Ferrari G, LaBranche C, Montefiori D, et al. 2017. An HIV envelope gp120-Fc fusion protein elicits effector antibody responses in rhesus macaques. Clin. Vaccine Immunol. 24.

8. Zhao B, Zhang X, Krummenacher C, Song S, Gao L, Zhang H, et al. 2018. Immunization with Fc-based recombinant epstein-barr virus gp350 elicits potent neutralizing humoral immune response in a BALB/c mice model. Front. Immunol. 9.

9. Zhang Y, Zhou Z, Zhu SL, Zu X, Wang Z, Zhang LK, et al. 2019. A novel RSV F-Fc fusion protein vaccine reduces lung injury induced by respiratory syncytial virus infection. Antiviral Res. 165: 11-22.

10. Kuo TT, Aveson VG. 2011. Neonatal Fc receptor and IgGbased therapeutics. MAbs 3: 422-430.
11. Zhao G, Du L, Ma C, Li Y, Li L, Poon VKM, et al. 2013. A safe and convenient pseudovirus-based inhibition assay to detect neutralizing antibodies and screen for viral entry inhibitors against the novel human coronavirus MERS-CoV. Virol. J. 10.

12. Pallesen J, Wang N, Corbett KS, Wrapp D, Kirchdoerfer RN, Turner HL, et al. 2017. Immunogenicity and structures of a rationally designed prefusion MERS-CoV spike antigen. Proc. Natl. Acad. Sci. USA 114: E7348-E7357.

13. Kebriaei A, Derakhshan M, Meshkat Z, Eidgahi MRA, Rezaee SA, Farsiani H, et al. 2016. Construction and immunogenicity of a new Fc-based subunit vaccine candidate against Mycobacterium tuberculosis. Mol. Biol. Rep. 43: 911-922.

14. Jung SY, Kang KW, Lee EY, Seo DW, Kim HL, Kim H, et al. 2018. Heterologous prime-boost vaccination with adenoviral vector and protein nanoparticles induces both Th1 and Th2 responses against Middle East respiratory syndrome coronavirus. Vaccine 36: 3468-3476.

15. Trivedi S, Miao C, Al-Abdallat MM, Haddadin A, Alqasrawi S, Iblan I, et al. 2018. Inclusion of MERS-spike protein ELISA in algorithm to determine serologic evidence of MERS-CoV infection. J. Med. Virol. 90: 367-371. 